\title{
Establishment of an in vitro monolayer model of macular corneal dystrophy
}

\author{
Sumit Murab, Shibu Chameettachal and Sourabh Ghosh
}

Macular corneal dystrophy (MCD) is characterized by multiple punctate gray-white opacities in the corneal stromal region, due to the accumulation of abnormally sulfated keratan sulfates. We attempted to develop an in vitro model of MCD by simulating the sulfation inhibition using sodium chlorate, a chemical inhibitor of 3'-phosphoadenosine-5'-phosphosulfate (PAPs). The SEM and micro-Raman spectroscopy results showed the hallmark feature of MCD. Further the gene expression studies elucidated the direct effect of sulfation inhibition on the WNT pathway, that in turn downregulated production of matrix metalloproteinases (MMPs), which causes abnormal matrix deposits leading to loss of transparency in vivo. It also resulted in downregulation of integrin and cadherin complexation that leads to disruption of the epithelial layer in the MCD affected corneas. This study offers a promising initial step toward establishing a relevant in vitro MCD disease model, to assess signaling transduction pathways and devise potential treatment strategies based on MMP administration to the MCD affected corneas.

Laboratory Investigation (2016) 96, 1311-1326; doi:10.1038/labinvest.2016.102; published online 17 October 2016

Macular corneal dystrophy (MCD) is the most common of the classical forms of inherited corneal dystrophies affecting stroma. ${ }^{1}$ MCD causes increasing corneal cloudiness resulting in progressive loss of vision. The endothelium, descemet's membrane and stroma get affected in the diseased state as the keratocytes and endothelial cells start accumulating intracytoplasmic vacuoles of abnormally sulfated keratan sulfate, ${ }^{2}$ but the epithelium is not involved in the degeneration process. Corneal thickness also gets reduced due to very dense packing of the collagen bundles as abnormally sulfated keratan sulfate molecules fail to form the bridges between the collagen bundles. $^{2}$

Despite the disease being one of the common causes of corneal blindness, there is no complete cure for it. Penetrating keratoplasty and deep anterior lamellar keratoplasty (DALK) are often done to treat the condition but the grafts again develop the haziness and thus fail. ${ }^{3}$ Penetrating keratoplasty offers an immediate improvement in the vision but is associated with a number of secondary complications. Although DALK offers much lesser complications but is associated with poor vision acuity and higher recurrence rates. ${ }^{3}$ The cause of MCD has been identified, but the pathophysiology of disease progression is not fully understood. Thus in order to understand the disease pathology and to develop related therapies and drugs; the development of an in vitro disease model system would be exceedingly necessary.
MCD is classified into two subtypes I and II, depending upon the presence or absence of sulfated keratan sulfate in the serum of the patients affected with the disease. ${ }^{4}$ Both the subtypes of the disease are caused due to a mutation in the gene Carbohydrate Sulfotransferase 6 (CHST6), which encodes the enzyme GlcNAc-6-sulphotransferase (C-GlcNAc6ST). MCD type I is caused by several mutations in the coding region of CHST6, while MCD type II is caused by deletions or replacements in the upstream region of CHST6 which causes complete inhibition of expression of CHST6 in the corneas. ${ }^{4}$ This mutation results in production of inactive form of C-GlcNAc6ST enzyme which is responsible for the sulfation of keratan sulfate molecules.

Keratan sulfate is the predominant glycosaminoglycan present in the corneal stroma. GAGs play a very important role in creating the regular lattice structure of collagen bundles in the corneal stroma which is responsible for the transparency of the tissue. The GAG molecules produce osmotic pressure as a result of the Donnan effect which helps in keeping the collagen bundles at specific distance that helps create the collagen lattice structure. The negatively charged sulfated side chains of these GAG molecules are basically responsible for creating this osmotic balance that keeps the corneal lattice intact. ${ }^{5}$ However, another theory suggests that the GAG molecules form duplex bridges between adjacent

Department of Textile Technology, Indian Institute of Technology Delhi, New Delhi, India

Correspondence: Dr S Ghosh, PhD, Department of Textile Technology, Indian Institute of Technology Delhi, Hauz Khas, New Delhi 110016, India.

E-mail: sghosh08@textile.iitd.ac.in

Received 26 July 2016; revised 13 August 2016; accepted 15 August 2016 
collagen bundle that help in maintaining the interfibrillar distance which is responsible for maintaining the regular corneal lattice structure. ${ }^{6-8}$ Taken together, both of these theories indicate the central role of sulfated GAGs in maintaining the corneal lattice structure, and in turn, the transparency. Thus, a disturbance in sulfation of keratan sulfate molecules would likely result in a disorder of the corneal lattice structure and loss of its transparency in both MCD types I and II.

The basic requirement for development of an in vitro disease model of MCD is the inhibition of sulfation of keratan sulfate molecules in the corneal stromal cells. In this study, sodium chlorate, which is a specific sulfotransferase inhibitor, was used to recapitulate the disease in vitro. Sodium chlorate inhibits $\quad 3^{\prime}$-phosphoadenosine- $5^{\prime}$-phosphosulfate (PAPS) synthetase- 2 which synthesizes PAPS. ${ }^{9}$ PAPS is the lone sulphur donor, thus inhibiting the enzyme which produces it; ceases the sulfation process of GAG molecules. ${ }^{9}$ Sodium chlorate has been earlier demonstrated to inhibit sulfation of proteoglycans during chondrogenesis. ${ }^{9}$ Sodium chlorate has also been reported for inducing desulfation of MG-63 osteosarcoma cells which negatively affected cell proliferation when exposed for short or medium duration. ${ }^{10}$ Long-term exposure of sodium chlorate hindered mineralization process, as it resulted in disruption of BMP2/noggin signaling pathway. ${ }^{10}$ Sodium chlorate at a concentration of $10 \mathrm{mM}$ was found to hinder $96 \%$ of sulfate incorporation in the proteoglycans of human fibroblasts. It also slightly affected the incorporation of leucine and glucosamine while there was no effect on degree of polymerization of the polysaccharide chain of the proteoglycans. ${ }^{11}$ Another study demonstrated the effect of sodium chlorate on sulfation of heparan sulfate in Madin-Darby canine kidney cells. ${ }^{12}$ Taken together, there are convincing evidences demonstrating role of sodium chlorate in specifically obstructing sulfation of proteoglycans by inhibiting PAPS synthetase activity. This property was exploited in the present study to design an in vitro disease model system of MCD.

The abnormally sulfated keratan sulfate molecules do not get degraded by the remodeling enzymes present in the corneal stroma. It will be interesting to know if these abnormally sulfated keratan sulfate molecules can be recognized by the matrix remodeling enzymes. Second, what is the effect of this undersulfation of keratan sulfate molecules on the underlying endothelial layer? Knowledge of such mechanisms will help us develop new clinical strategies to tackle MCD without wasting the already scarce donor corneal tissue because of recurrence of the opacity.

Thus in the present study we have attempted to simulate the in vivo conditions prevailing in MCD by inhibiting the sulfation of keratan sulfate in corneal stromal cells (keratocytes). This study can pave the way for more advanced model systems for the study of pathophysiology of this disease and can also be used for the search of new molecules and clinical strategies for the treatment of this disease that makes a major share in inducing corneal blindness. This study for the first time demonstrated the effect of inhibition of sulfation of keratan sulfate in corneal stromal cells; mimicking the physiological conditions prevailing in MCD affected corneas.

\section{METHODS AND MATERIALS Corneal Stromal Cell Isolation}

Cadaveric goat eyes were collected from All India Institute of Medical Sciences, New Delhi, India with prior approval from the Institute Ethical Committee. The goat corneas were excised from the ocular globe under sterile conditions and washed several times in PBS (Himedia Laboratories; pH 7.27.4) containing $100 \mathrm{U} / \mathrm{ml}$ penicillin- streptomycin (Lonza, USA), $50 \mu \mathrm{g} / \mathrm{ml}$ gentamycin (Himedia Laboratories), and $100 \mu \mathrm{g} / \mathrm{ml}$ amphotericin B (Himedia Laboratories; antibiotic mixture).

Goat cornea was isolated from the ocular globe by removing the sclera, soft connective tissue and the limbal rings. After separating the three layers carefully, the stromal layer was cut into small pieces of $\sim 2-3 \mathrm{~mm}$ and washed extensively with antibiotic mixture. After thoroughly rinsing with PBS, the fragmented tissue pieces were carefully lifted using sterile forceps and incubated at the bottom of a T25 tissue culture flask for 7 days in DMEM (CELLclone, India). The freshly isolated goat corneal stromal cells were expanded in DMEM with $10 \%$ fetal bovine serum (Biological Industries, India) and $100 \mathrm{U} / \mathrm{ml}$ penicillin-streptomycin. For serial passaging, cells were washed with PBS and dissociated using $0.25 \%$ trypsin $(\mathrm{v} / \mathrm{v}$; Lonza, USA) diluted in PBS. Standard culture conditions of $5 \% \mathrm{CO}_{2}$ and $95 \%$ humidity at $37^{\circ} \mathrm{C}$ were maintained.

\section{Corneal Stromal Cell Culture with Sodium Chlorate}

Corneal stromal cells were cultured on coverslips in 24-well plates with an initial seeding density of $1 \times 10^{5}$ cells/well. Alpha MEM with $10 \%$ fetal bovine serum (Himedia Laboratories, Mumbai, India), 1\% penicillin-streptomycin (Himedia Laboratories), 1\% amphotericin (Himedia Laboratories), $0.5 \%$ gentamicin (Himedia Laboratories), and $1 \%$ HEPES (Himedia Laboratories) was used for culturing the cells. Sodium chlorate (Sigma-Aldrich, Bengaluru, India) was added to the culture media at a concentration of 5,10 , and $50 \mathrm{mM}$ for three respective test groups while the control group was cultured without sodium chlorate. Samples for different studies were collected at day 1,7 , and 14 . The groups have been coded as given in Table 1 that has been used throughout the manuscript.

\section{MTT Assay}

MTT assay was used to assess the metabolic activity of the corneal stromal cells at days 1, 7, and 14. For the assay, cells were incubated in a 9:1 ratio of standard culture media and MTT (3-(4,5-dimethylthiazol-2-yl)-2, 5-diphenyl tetrasodium bromide, Millipore CT0-A, USA) and incubated for $6 \mathrm{~h}$ at $37^{\circ}$ C. Following incubation, the media-MTT solution was 
Table 1 Code name for the three test and one control groups

\begin{tabular}{lr}
\hline Group & Code \\
\hline $5 \mathrm{mM}$ Sodium chlorate & 5 \\
$10 \mathrm{mM}$ Sodium chlorate & 10 \\
$50 \mathrm{mM}$ Sodium chlorate & 50 \\
Without sodium chlorate & w/o \\
\end{tabular}

centrifuged to collect the insoluble formazan crystals which were solubilised in $1 \mathrm{ml}$ DMSO to dissolve the crystals. Absorbance was measured at $560 \mathrm{~nm}$ using microplate reader (BIORAD iMark Microplate reader, USA). Standard calibration curve was made by plotting the absorbance values obtained for the test samples (normalized to control) against the counted cell numbers.

\section{DNA Quantification}

DNA was isolated from the cultured corneal stromal cells using DNA extraction kit (Agilent Technologies, Germany, Catalog no. 200600-1) according to the manufacturer's guidelines. Quantification was performed on a spectrophotometer (Thermo Scientific NanoDrop 2000c, USA).

\section{Biochemical Estimation}

Corneal stromal cells were analyzed for GAG and collagen production after 14 days of culture and the values were normalized to their DNA content. For biochemical analysis, the constructs were digested with proteinase $\mathrm{K}$ (SRL, Mumbai) at $60^{\circ} \mathrm{C}$ overnight. Portions of the digest were then analyzed to quantify sulfated GAG and hydroxyproline content. GAG content was determined by dimethylmethylene blue assay using chondroitin sulfate (Himedia Laboratories) calibration curve. ${ }^{13}$ The hydroxyproline content was converted to total collagen content using a 7.25 mass ratio of collagen to hydroxyproline. The spectrophotometric readings were taken on an iMark Microplate Absorbance Reader, BIORAD.

\section{Scanning Electron Microscopy}

For scanning electron microscopy (SEM) analysis, all samples were vacuum dried and coated with gold using a gold sputter coater (EMITECH K550X, UK) at $25 \mathrm{~mA}$ for $1 \mathrm{~min}$, to form a coating of $\sim 15-20 \mathrm{~nm}$ thickness. Cellular morphology was imaged using SEM at an accelerating voltage of $20 \mathrm{kV}$ (Zeiss EVO 50). Cellular morphology was monitored on the coverslips seeded with corneal stromal cells on days 1 and 14 .

\section{Raman Analysis of ECM}

Raman spectra of the extracellular matrix was obtained using a confocal laser dispersion Micro-Raman spectrometer (inVia reflex, UK) system equipped with a $785 \mathrm{~nm}$ diode laser and integrated with FTIR IlluminatIR II module. ${ }^{14}$ This system was combined with Leica microscope to allow scatter, line, area mapping, and confocal depth profiling. All Raman measurements were taken at $\times 10$ magnification. The samples were placed on aluminium slides and measurements were taken on five randomly selected points per sample with an integration time of $10 \mathrm{~s}$.

\section{Histochemistry and Immunofluorescence Studies}

After 14 days of culture, cells were fixed with $10 \%$ formaldehyde for $4 \mathrm{~h}$, washed with PBS twice. The cells were then stained with hematoxylin and eosin (H\&E) to check cellular morphology. Safranin-O and alcian blue staining were used to visualize the extracellular GAG accumulated on the scaffold. Cell samples were blocked by $10 \%$ bovine serum albumin for $30 \mathrm{~min}$ at room temperature, and incubated with primary antibodies against Collagen type I (ab90395/Abcam), Cytokeratin 14 (KRT 14) (ab7800/Abcam), Fibronectin-1 (FN 1) (ab6328/Abcam), E-cadherin (E-CD) (ab1416/Abcam), Actin (ACT) (PF7551/ECM Biosciences). and 4',6'-diamidino-2-phenylindole (DAPI/32670/Sigma Aldrich) was used to stain the nucleus. Alexa Fluor 546 goat anti-mouse IgG (1:200, Invitrogen) was used for fluorescent labelling. The sections were given three consecutive washed with PBS. Following this step, specimens were rinsed twice with PBS/ Tween and the sections were subsequently mounted onto slides. Immunostained sections were examined using Confocal Laser Scanning Microscope (Leica TCS SP5, Germany).

\section{Digital Image Analysis}

For image analysis, five randomly selected regions from immunohistochemistry micrographs were analyzed independently for each respective sample; one representative image has been shown. Color de-convolution technique was used as described earlier for the analysis. ${ }^{15}$ Pure safranin-O/alcian blue staining patterns were assigned with an automated score after observation and measurement of every histogram profile generated by Image $\mathrm{J}$ software (NIH, USA). The pixel numbers of particular intensity were represented against corresponding intensity by the histogram profile. Thus, categorization was made into high positive, positive, and negative zones depending on the color score intensity of the images.

\section{Quantitative Real-Time Reverse Transcription Polymerase Chain Reaction}

All cell samples were disrupted in Trizol (Invitrogen) and total mRNA was extracted as per the manufacture's protocol. The aqueous phase was transferred to an RNeasy mini-spin column (Qiagen, Germany), eluted and then the RNA samples were reverse transcribed into cDNA according to the manufacture's protocol. The expression of COL 1 (QT00037793), biglycan (QT00000483), MMP 1 (QT000 14581), MMP 13 (QT00001764), RAC 1 (QT00065856), RHO A (QT00044723), SMAD 4 (QT00013174), $\beta$-catenin $(\beta-\mathrm{CTNN}) \quad(\mathrm{QT} 00077882), \quad \mathrm{KRT}-14 \quad(\mathrm{QT} 00052283), \quad \mathrm{FN}$ 1 (QT00038024), CDH 1 (QT00080143), and GAPDH 
(QT00079247) were quantified using QuantiTect primer assay (Qiagen) and Rotorgene real-time PCR system (Qiagen, Germany), and were normalized against glyceraldehyde-3phosphate-dehydrogenase (GAPDH) (QT00079247). The analysis was performed using Rotorgene Q software and the relative expression levels were calculated using $2^{-(\Delta \Delta c(t))}$ method. ${ }^{16}$

\section{Protein-Protein Interaction and Gene Ontological Studies}

The protein-protein interactions were studied using a database of known and predicted protein interactions STRING 10.0 (Search Tool for the Retrieval of Interacting Genes/Proteins) software (http://string-db.org). Although the gene ontological studies were performed by using PANTHER gene ontological analysis server (http://pantherdb.org). ${ }^{17}$

\section{Statistical Analysis}

Data have been presented as mean \pm s.d. The number of replicates has been indicated with the respective methods. Single factor paired one-tailed Student's $t$-test was used for the analysis of the significance of variations in loading efficiency. Two-way ANOVA followed by Fischer's post hoc analysis was used for the analysis of the significance of the variations for all other experiments. The difference between different test and control groups was calculated by $P$-value. The groups with $P<0.05$ were considered as statistically significantly different.

\section{RESULTS}

\section{Metabolic Activity}

The cellular metabolic activity (Figure 1a) on day 1 after seeding (without the addition of sodium chlorate) was comparable in all the three test groups and the control set. Although after addition of sodium chlorate on day 2, the metabolic activity showed a marked difference; when checked on day 7. The metabolic activity of the corneal stromal cells decreased as a function of increasing sodium chlorate concentration. The highest metabolic activity was observed for the control set. This suggests the concentration-dependent inhibitory effect of sodium chlorate on the metabolic activity of corneal stromal cells. Further on day 14, the metabolic activity of all the test groups and the control set again became comparable with an overall increase over the day 7 values. This indicates that the corneal stromal cells became acclimatized to the inhibitor, sodium chlorate after about 10 days of addition into the culture.

\section{Cell Proliferation}

DNA content (Figure 1b) was estimated to analyze the cellular proliferation under the influence of different concentrations of sodium chlorate. All the test groups and the control showed no statistically significant difference in DNA content, which signifies that there was nominal effect of sodium chlorate on cellular proliferation at day $14(P<0.01, n=3)$.

\section{Biochemical Estimation}

The total GAG content was found to be $647.34 \pm 4.5$, $646.83 \pm 3.2,431.53 \pm 2.1$, and $701.03 \pm 8.7 \mathrm{ng} / \mathrm{ng}$ for 5,10 , $50 \mathrm{mM}$, and the control group, respectively $(P<0.01, n=3$; Figure 1c). The increasing concentration of inhibitor showed a significant detrimental effect on GAG production at $50 \mathrm{mM}$ as compared with $5 \mathrm{mM}, 10 \mathrm{mM}$, and the control group. The total collagen content (Figure 1d) in all the samples was lower as compared with total GAG content but the effect of inhibitor was much more pronounced on total collagen content. The total collagen content was found to be $0.62 \pm 0.04,0.52 \pm 0.01,0.29 \pm .03$, and $1.38 \pm 0.03 \mathrm{ng} / \mathrm{ng}$ for $5,10,50 \mathrm{mM}$, and the control group, respectively $(P<0.01$, $n=3)$.

\section{Cellular Morphology}

The corneal stromal cells (Figure $2 \mathrm{a}-\mathrm{j}$ ) on day 1 after addition of sodium chlorate were observed. The cells with $5 \mathrm{mM}$ of sodium chlorate showed spread morphologies with deposition of bright crystal like extracellular matrix, while the cellular morphology became more rounded with increasing concentration of sodium chlorate in the media. Also, the presence of white crystal like extracellular deposits decreased with the increasing concentration of sodium chlorate. The control group cells without sodium chlorate showed spread morphologies with the extensive deposition of white crystalline extracellular matrix which evenly covered the cellular surface. This white crystalline extracellular matrix was probably keratan sulfate produced by the corneal stromal cells.

At day 14, increased deposition of white crystalline extracellular matrix in all the test group and control was observed. The morphology though changed from rounded to stretched which is a hallmark of stressed cells; in all the test groups. In the $50 \mathrm{mM}$ group, large aggregates of possibly abnormally sulfated GAGs were visible; which is a hallmark of the disease and results into corneal opacity. On the other hand, the control group (without sodium chlorate) showed high deposition of non-aggregated white crystalline extracellular matrix.

\section{Micro-Raman Spectroscopic Analysis}

Micro-Raman spectrophotometery was used to validate the nature of the white crystal like extracellular matrix deposits seen in SEM images (Figure $2 \mathrm{k}$ ). The spectra clearly showed a prominent peak at around 1009 per $\mathrm{cm}$ in all the samples, which is attributed to the sulfate group. ${ }^{18}$ The intensity of the peak decreased as a function of increasing concentration of sodium chlorate showing its direct effect on the sulfation of keratan sulfate which is the predominant glycosaminoglycan molecules present in corneal stroma. This confirmed the chemical nature of the white granular extracellular deposits seen in the SEM images as keratan sulfate. This observation also validated the utility of sodium chlorate in establishing a disease model system of corneal macular dystrophy by 

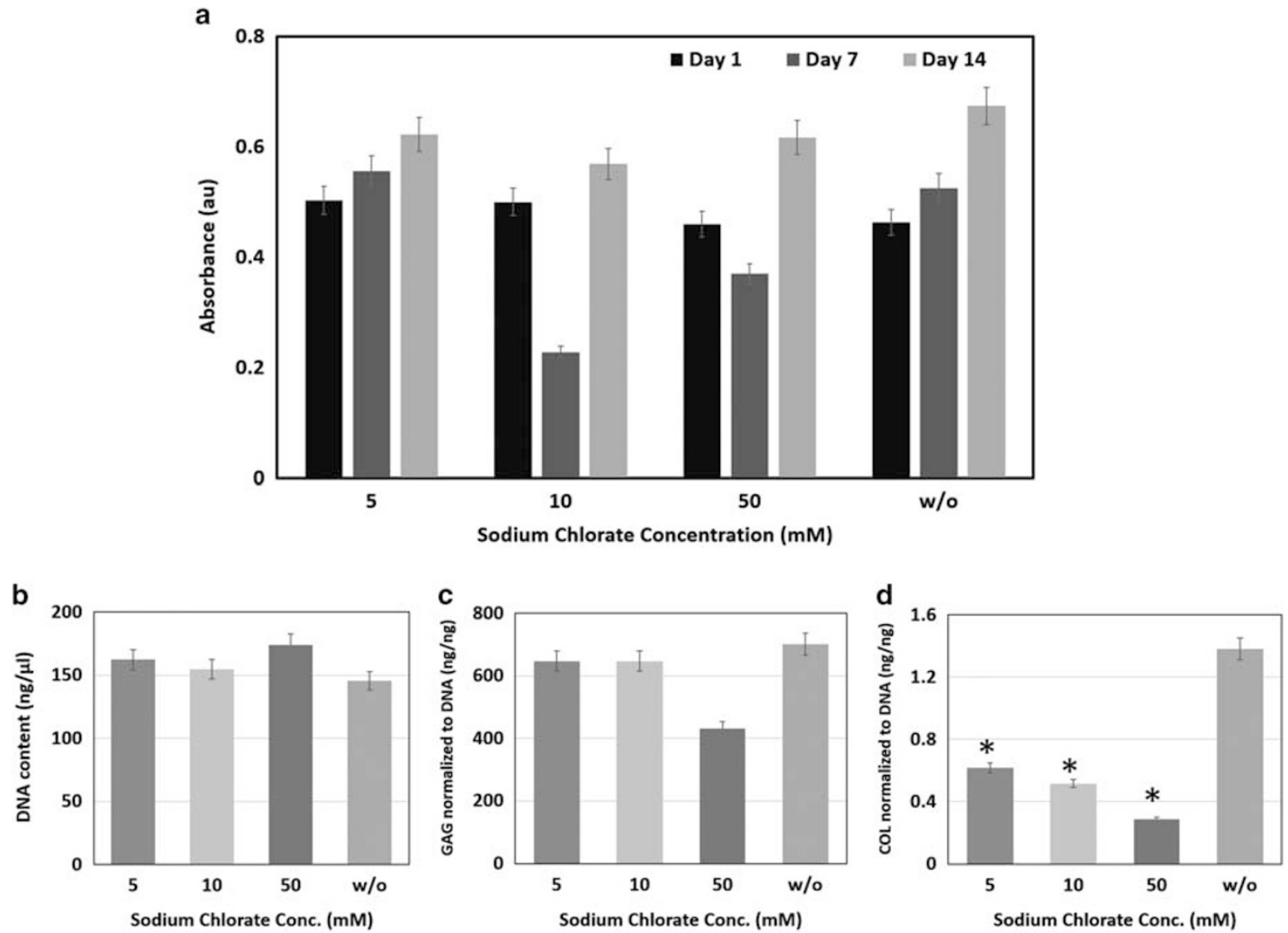

Figure 1 (a) MMT assay of corneal stromal cells at days 1, 7, and 14 showing their respective metabolic activities. The metabolic activity was hindered after addition of sodium chlorate initially as seen in day 7 data, but it resumed to baseline later (day 14). (b) Total DNA content of the corneal stromal cells containing 5, 10,50 mM, and no sodium chlorate at day 14. This indicated that sodium chlorate did not hinder the proliferation of keratocytes as all the groups had similar DNA content. (c) Total GAG content of the four groups normalized to their respective total DNA contents at day 14. (d) Total collagen content of the four groups normalized to their respective total DNA contents at day 14 (statistical significance between tests and control $\left.{ }^{*} P<0.01, n=3\right)$.

quantitatively controlling the sulfation of keratan sulfate, which is the hallmark of the disease.

\section{Histology}

\section{$H$ \& E staining}

$\mathrm{H} \& \mathrm{E}$ staining (Figure 3a-d) on visual examination showed no changes in cell numbers between the different groups, as also evident from the MTT readings at day 14. There was sparse matrix deposition in all the test samples and also the control group. But the morphologies of the cells showed clear effect of the increasing concentration of sodium chlorate. With increasing concentration of sodium chlorate the cells became stretched while the control group cells demonstrated spread morphologies.

\section{Safranin-O and Alcian Blue Staining}

Safranin-O (Figure 4a-d) and alcian blue (Figure 5a-d) staining were carried out to visualize the sulfated GAGs produced by the corneal stromal cells. Both the staining demonstrated decreased deposition of sulfated GAGs as a function of increasing concentration of sodium chlorate, while the control group showed significant deposition of sulfated GAGs. The histological images were quantified for staining using digital image analysis. In both the staining protocols; with the increase in sodium chlorate concentration the staining intensity decreased toward the low positive side (score: 255) from the higher positive side (score: 0 ). This was in accordance with the biochemical quantification of sulfated GAGs and further validated the use of sodium chlorate in establishment of a biochemical model of corneal macular dystrophy by quantitatively controlling the sulfation of keratan sulfate molecules.

\section{Gene Expression Analysis}

The gene expression studies (Figure 6a-k) were carried out to assess the impact of inhibition of sulfation of keratan sulfate on corneal stromal cells. The expression of corneal stromal marker Cytokeratin 14 (KRT 14) was suppressed significantly $(P<0.001, n=3)$ even by $5 \mathrm{mM}$ concentration of sodium chlorate. Higher concentrations of 10 and $50 \mathrm{mM}$ resulted in downregulation of this marker. Fibronectin, an extracellular matrix glycoprotein which is engaged in cellular attachment 

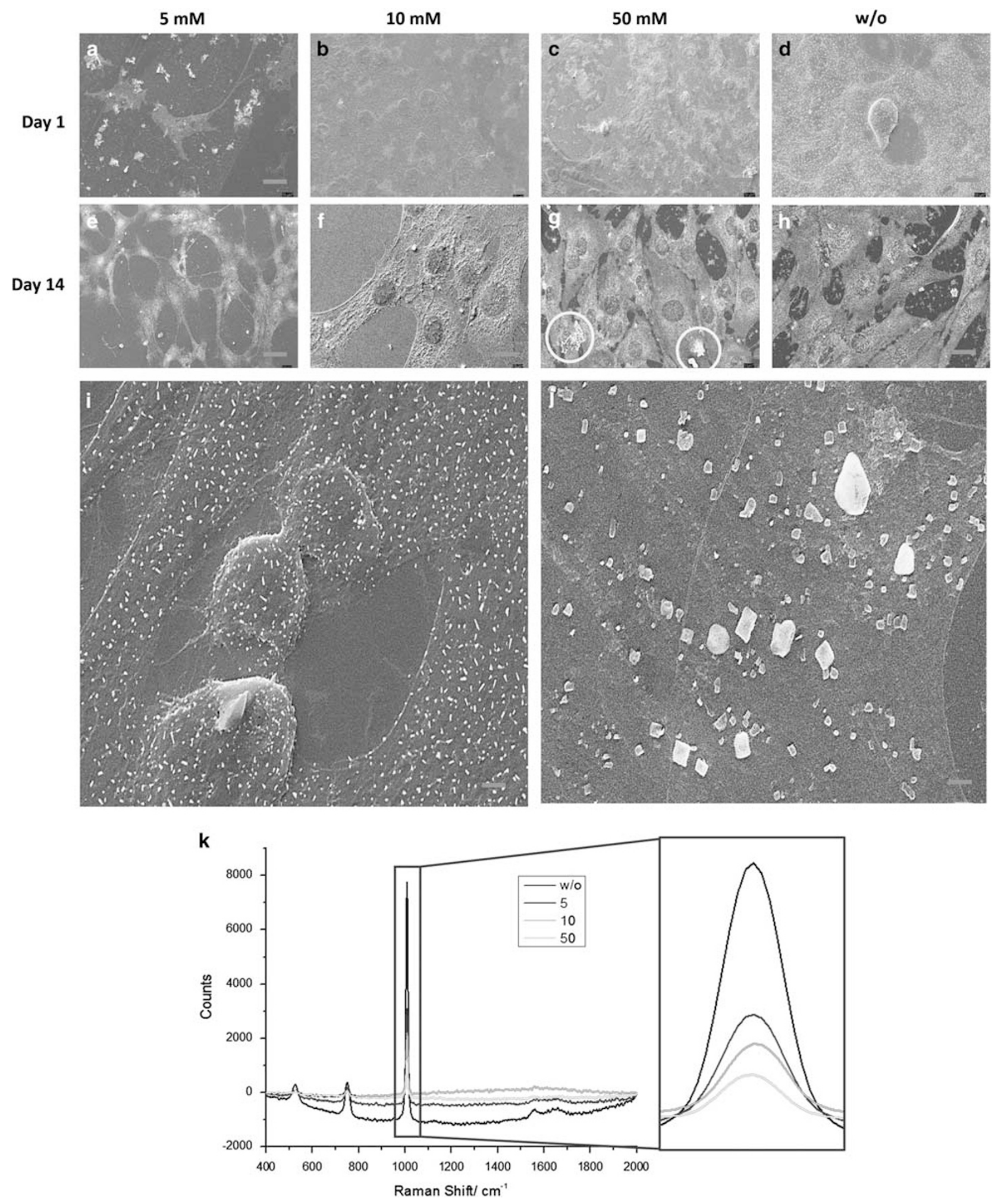

to the matrix, also demonstrated significant downregulation $(P<0.001, n=3)$ in the presence of sodium chlorate as compared with the control group. But the effect of increasing inhibitor concentration was not observed in this case. E-cadherin, which is responsible for cell to cell attachments, was also significantly inhibited in the presence of sodium chlorate. A clear effect of increasing inhibitor concentration was observed in this case; $5 \mathrm{mM}$ concentration decreased the expression of E-cadherin; while 10 and $50 \mathrm{mM}$ completely inhibited its expression. Extracellular proteins namely 
Figure 2 SEM micrographs of corneal stromal cells treated with 5, 10, 50 mM, and no sodium chlorate at day 1 (a-d) and day 14 (e-h), respectively (scale bar, $10 \mu \mathrm{m}$ ). In Figure 1g, the circles highlight the aggregates of abnormally sulfated keratan sulfate that are the hallmark of MCD and causes loss of transparency of corneas. Magnified images of the keratan sulfate crystals deposited by the corneal stromal cells in the control group: (i) scale bar, $3 \mu \mathrm{m}$, (j) scale bar, $1 \mu \mathrm{m}$. (k) Micro-Raman spectra of the four groups with $5 \mathrm{mM}, 10 \mathrm{mM}, 50 \mathrm{~mm}$, and no sodium chlorate. The spectra show a clear peak of sulfate at 1009 per $\mathrm{cm}$. The peak intensity decreases with the increasing concentration of sodium chlorate in the culture demonstrating a clear inhibition of sulfation process in the corneal stromal cells due to sodium chlorate (inset shows a zoomed view of the four peaks for comparison).
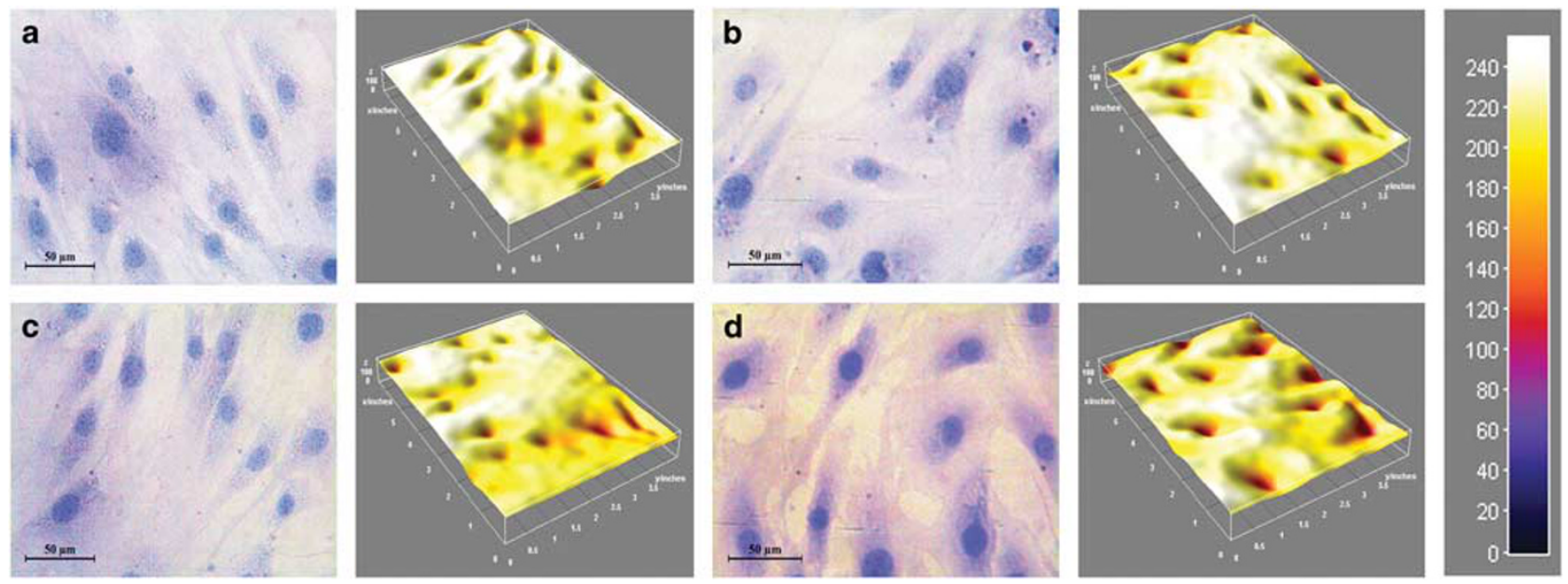

Figure 3 H\&E staining of the corneal stromal cells at day 14 with (a) $5 \mathrm{mM}$, (b) $10 \mathrm{mM}$, (c) $50 \mathrm{mM}$, and (d) no sodium chlorate. Scale bar, $50 \mu \mathrm{m}$ (a-d). The increasing concentration of sodium chlorate showed a marked effect on the morphology of the corneal stromal cells as the cells started stretching with its increasing concentration. The matrix staining also decreased with the increasing concentration of sodium chlorate showing its negative effect on matrix production.

biglycan and collagen type I also demonstrated a similar trend of inhibitor concentration on their expression patterns. Biglycan, which is a core protein of proteoglycans, demonstrated a downregulation in its expression as a result of $5 \mathrm{mM}$ inhibitor concentration, while higher concentrations of 10 and $50 \mathrm{mM}$ inhibited its expression. COL I though showed a similar trend but its expression ceased at $50 \mathrm{mM}$ concentration. RHO A and RAC 1 genes also showed inhibition because of sodium chlorate. The expression of RHO A decreased as a function of increasing inhibitor concentration, while RAC 1 showed a downregulation at all concentrations but not as a function of increasing inhibitor concentration. Fibronectin showed a concentration independent inhibition due to sodium chlorate while KRT 14 and E-cadherin both demonstrated a concentration-dependent gene expression inhibition. Both the genes ceased to express at 10 and $50 \mathrm{mM}$ concentrations of sodium chlorate. To analyze the signaling pathways involved in the effect of sodium chlorate on corneal stromal cells, SMAD 4 from the SMAD-MAPK pathway and $\beta$-catenin from the WNT pathway were studied. SMAD 4 showed inhibition of its expression due to sodium chlorate but the expression was not a function of the increasing inhibitor concentration. Although $\beta$-catenin expression decreased as a function of increasing sodium chlorate concentration demonstrating a direct involvement of WNT pathway in the detrimental action caused by sodium chlorate on corneal stromal cells.

\section{Immunohistochemistry}

Immunohistochemistry (Figure 7a-e) and subsequent fluorescence quantification (Figure $8 \mathrm{a}-\mathrm{d}$ ) was performed. Immunohistochemical staining confirmed the change in morphology observed as a function of increasing sodium chlorate concentration. The stretched actin cytoskeleton was noticed a function of increasing sodium chlorate concentration. The control group without sodium chlorate showed cells with relaxed actin cytoskeleton and spread morphologies, thus validating the H\&E results. The immunostaining results showed minimum expression of Collagen type I in all the test groups and control, validating the gene expression studies and biochemical estimation. Cytokeratin 14 expression was decreased as a function of increasing concentration of sodium chlorate. In contrast, the control group of cells showed significant $(P<0.001, n=3)$ expression of Cytokeratin 14 protein. E-Cadherin showed a diffused expression throughout the cellular surface in the control group. The test groups showed decreased expression of E-Cadherin as a function of increasing concentration of sodium chlorate. The expression pattern also became localized as longitudinal patches that may indicate the stress-induced changes in cellular attachment; because of sodium chlorate. Fibronectin showed a peculiar 

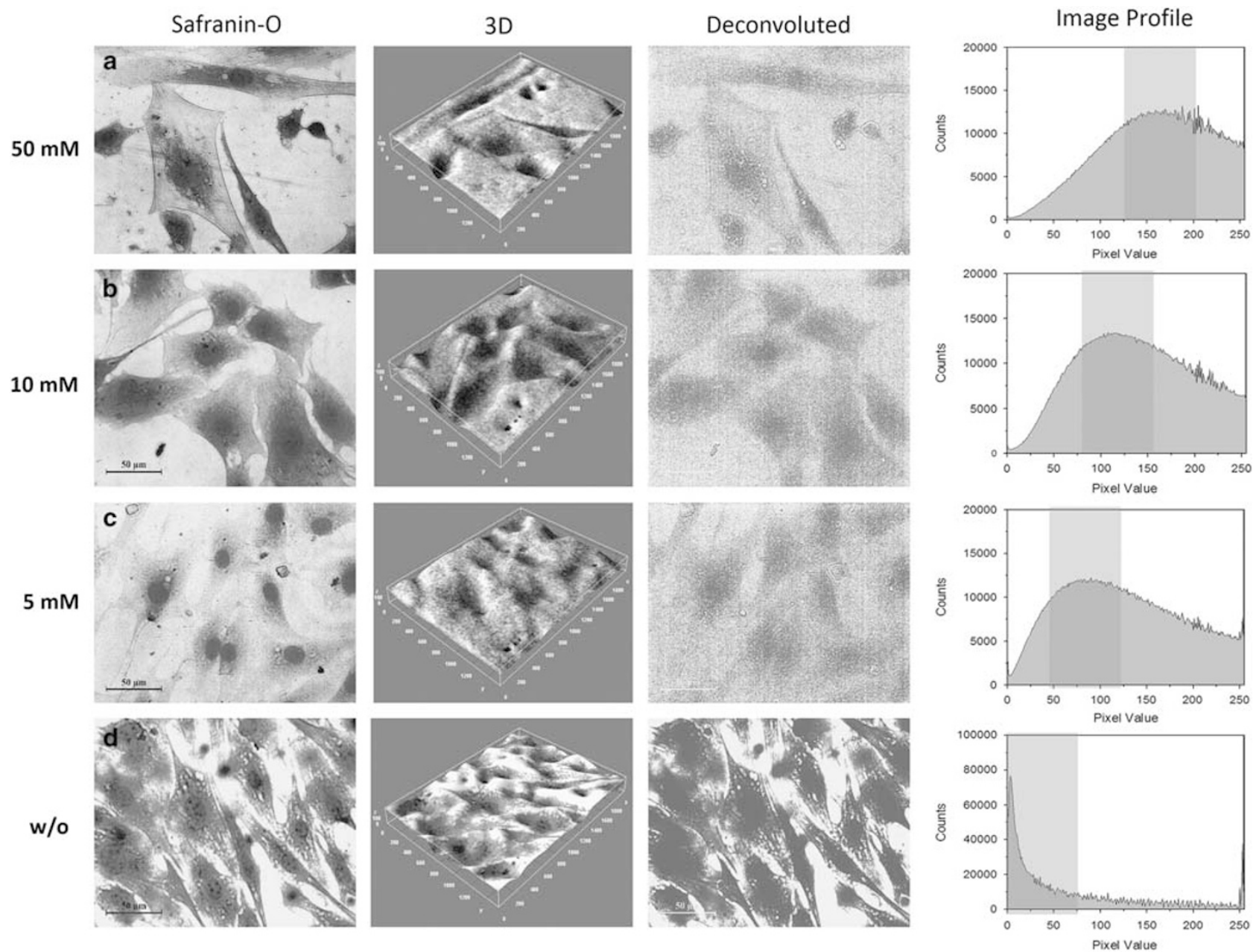

Figure 4 Histochemistry of the cell laden hydrogels after 14 days of corneal stromal cell culture: Safranin-O staining, 3D surface plot of stained images showing deposition and distribution of GAG, color de-convoluted image, reference histogram profile: (a) $5 \mathrm{mM}$, (b) $10 \mathrm{mM}$, (c) $50 \mathrm{mM}$, and (d) no sodium chlorate. Scale bar, $50 \mu \mathrm{m}(\mathrm{a}-\mathrm{d})$. 3D image reconstruction and software-based analysis data set of constructs representing score was done by using Image $\mathrm{J}(\mathrm{NIH})$ software by thresholding of stained zones of IHC images followed by pixel vs intensity determination by color de-convolution plugin.

expression pattern; the protein was evenly expressed all over the cellular surface in the control group while the expression became localized to the periphery of the cells with increasing concentration of sodium chlorate from 5 to $10 \mathrm{mM}$. The $10 \mathrm{mM}$ group showed prominent localized expression of fibronectin. But the protein expression drastically reduced in the $50 \mathrm{mM}$ group showing cessation of expression of fibronectin at this concentration.

\section{Protein-Protein Interaction Analysis}

The protein-protein interaction analysis (Figure 9a) demonstrated a high degree of interaction between the genes taken up for gene expression study. SMAD 4 showed interactions with five other genes (COL I, FN 1, RAC 1, RHO A, and $\mathrm{CDH} 1$ ); showing its involvement in the physiology of the diseased condition. It also confirmed the results from the gene expression analysis that showed a direct relation of sodium chlorate concentration on expression of these genes which may be coordinated by SMAD 4. Although $\beta$-catenin was found to form the biggest subnetwork with highest protein interactions taking place from this hub. It interacted with $\mathrm{RHO} \mathrm{A}, \mathrm{RAC} 1$, and $\mathrm{CDH} 1$ genes demonstrating its involvement in the disease pathology through disruption of cellular interactions with other cells and matrix. This also validated the expression behavior of $\beta$-catenin gene which was dependent on the concentration of sodium chlorate. KRT-14 was linked to $\mathrm{CDH} 1$ (E-Cadherin) that validated the trend observed in expression of both the genes, higher concentrations (10 and $50 \mathrm{mM}$ ) of sodium chlorate ceased the expression of both. Biglycan and COL I were found to be linked by three evidences validating the similar gene expression profiles of these two genes.

\section{Gene Ontological Analysis}

Gene ontological analysis (Figure 9b) demonstrated the involvement of 24 signaling pathways on the basis of the 11 

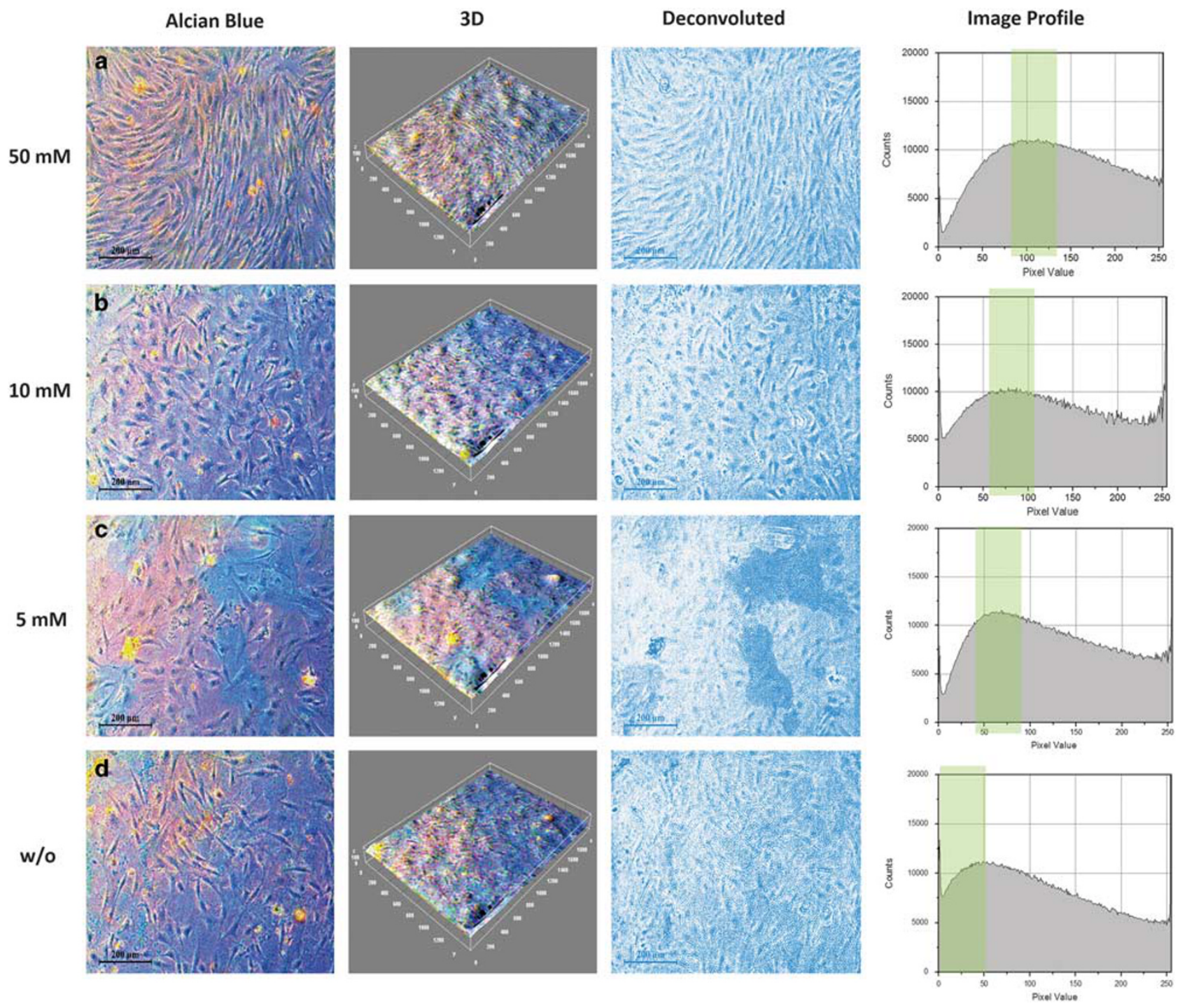

Figure 5 Histochemistry of the cell laden hydrogels after 14 days of corneal stromal cell culture: Alcian Blue, 3D surface plot of stained images showing deposition and distribution of GAG, color de-convoluted image, reference histogram profile: (a) $5 \mathrm{mM}$, (b) $10 \mathrm{mM}$, (c) $50 \mathrm{mM}$, and (d) no sodium chlorate. Scale bar, $50 \mu \mathrm{m}(\mathrm{a}-\mathrm{d})$. 3D image reconstruction and software-based analysis data set of constructs representing score was done by using Image $\mathrm{J}(\mathrm{NIH})$ software by thresholding of stained zones of IHC images followed by pixel vs intensity determination by color de-convolution plugin.

genes studied in the present work. The gene ontological pathway analysis showed the involvement of WNT, integrin, cadherin, and cytoskeletal signaling pathways in the disease progression of MCD. This demonstrated the possible involvement of WNT pathway in the pathophysiology of the disease. Integrin signaling pathway was involved with the highest number of genes $(n=4)$ that showed the association of cellular attachment that may result in the disruption of the endothelial layer of diseased cornea.

\section{DISCUSSION}

The onset of MCD may have a genetic origin, but enhanced perception about its progression and implications on the tissue structure, biochemistry, and function can help to understand the disease better and device strategies for its clinical treatment and development of drugs or other therapies. In diseases like MCD where the disease pathophysiology is relatively unknown, the development of an in vitro disease model system can pave the way for gaining insight into the mechanism of progression of the disease. ${ }^{16}$

MCD is caused due to an autosomal recessive disorder in CHST6 gene of the chromosome 16 (16q22). ${ }^{4}$ The disease pathology begins with the deposition of abnormally sulfated proteoglycans which consists of unsulfated or partially sulfated keratan sulfate molecules, in the corneal stroma, Descemet's membrane and Bowman's layer. Endoplasmic reticulum of keratocytes have been reported to be filled with these abnormally sulfated keratan sulfate that fail to be secreted out in the extracellular matrix. ${ }^{2,19,20}$ Electron-lucent vacuoles can be visible all over the corneal stroma in $\mathrm{MCD}$ 

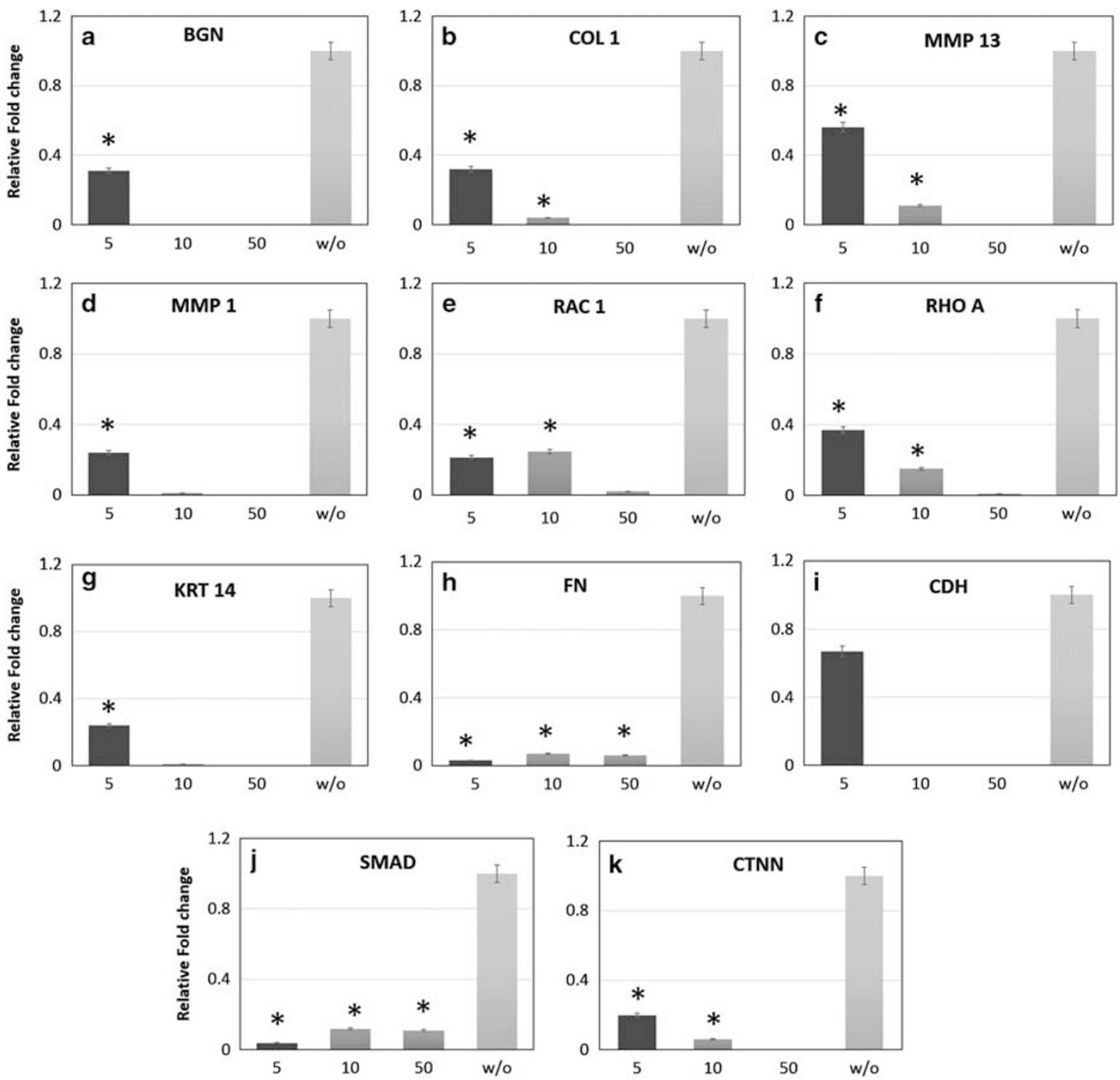

Figure 6 Quantitative Real-time PCR studies to evaluate gene expression in corneal stromal cells cultured with 5, 10, 50 mM, and no sodium chlorate at day 14: (a) biglycan, (b) COL 1, (c) MMP13, (d) MMP 1, (e) RAC 1, (f) RHO A, (g) KRT 14, (h) fibronectin, (i) cadherin, (j) SMAD 4, and (k) CTNN $\beta 1$ (statistical significance between tests and control: $P<0.01, n=3$ ). All the genes taken for this study showed downregulation in their expression due to sodium chlorate.

affected corneas, sometimes filled with abnormally sulfated keratan sulfate proteoglycan filaments. ${ }^{21}$ Structurally, the collagen lattice is disturbed. Though the collagen fibers have normal diameter but the interfibrillar spacing is reduced as compared with normal corneas, which results in reduction of the overall thickness of MCD affected corneas. ${ }^{22}$ This close packing is probably due to the absence of proteoglycan bridges between the collagen fibrils that maintain the corneal lattice structure as the sulfated chain is absent in keratan sulfate of MCD affected corneas. The Descemet's membrane of MCD affected corneas is normal in the anterior portion but the posterior portion of the membrane develops corneal lesions and electron-lucent vacuoles. ${ }^{2,19}$ It has also been reported that apart from the decrease of interfibrillar distance; as we proceed from anterior to posterior part of cornea, the presence of large diameter collagen fibrils increases that probably also has a role in loss of corneal transparency. ${ }^{23}$ This indicates a major role of endothelium in producing these abnormalities in the stroma as the posterior part of stroma is severely affected by the abnormalities caused by the mutation in the sulfotransferase gene (CHST6). Descemet's membrane is the true basement membrane secreted by the endothelium. Thus the abnormal appearance and adenosine monophosphate deposits may be derived from the endothelium of the corneal tissue. The anterior portion of MCD-affected corneas is laid down during fetal development, so the ultrastructure of this portion is normal as opposed to the lesions of the posterior portion. This helps in explaining the development 

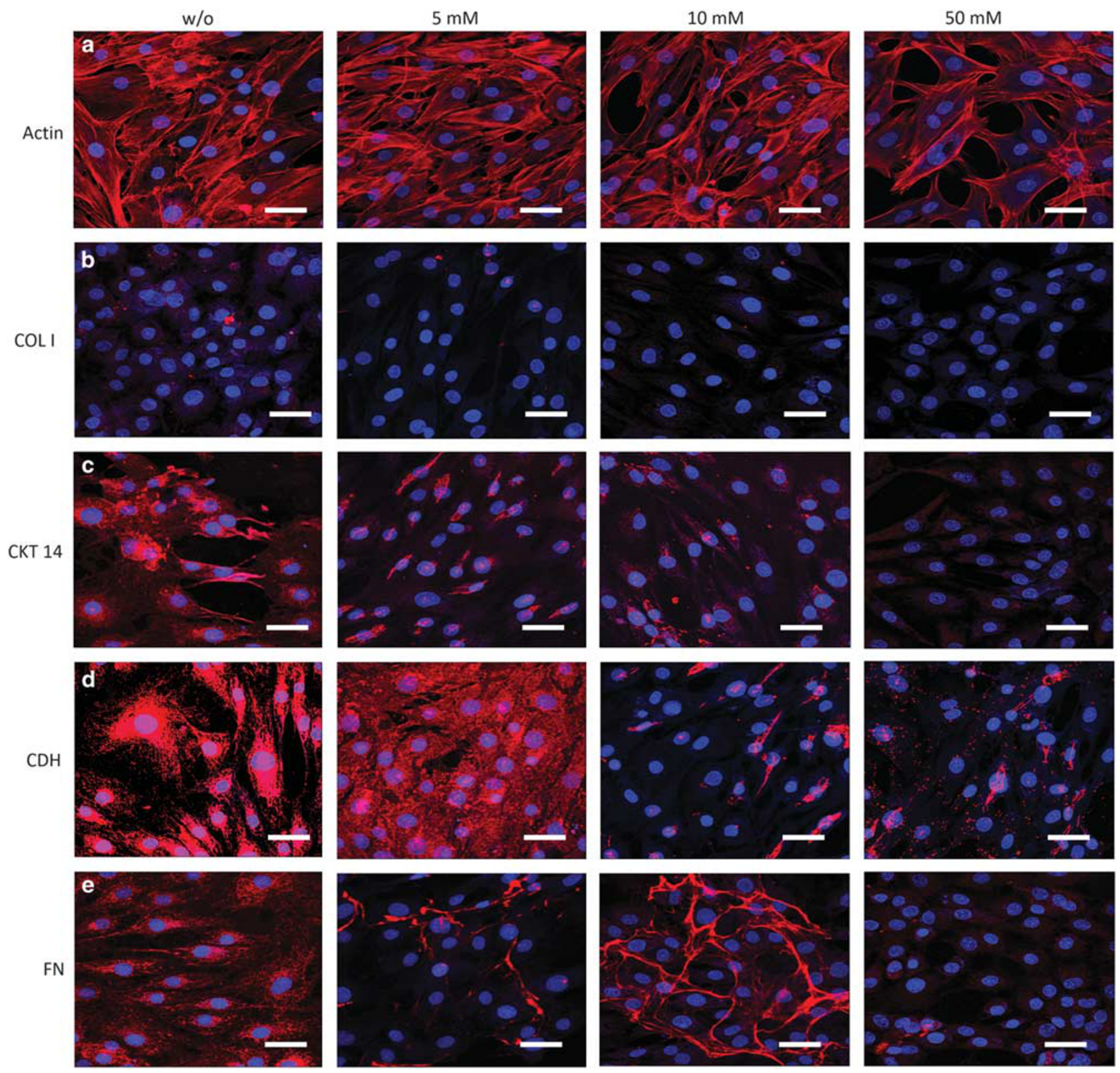

Figure 7 Immunohistochemical analysis of corneal stromal cells after 14 days of culture with 5, 10,50 mM, and no sodium chlorate: (a) actin, (b) COL I, (c) KRT 14, (d) CDH 1, and (e) FN 1. Scale bar, $50 \mu \mathrm{m}$ (a-e). Sections were stained with actin, COL I, KRT 14, CDH 1 and FN 1 antibodies (red) and counterstained with DAPI (blue) for cell nuclei.

of disease during the later portion of the patient's life, not congenitally. This highlights a basic difference between the physiology of adult and fetal endothelial cells. It can also be due to the conditions prevailing during these two periods that causes expression of differently sulfated keratan sulfate molecules at different time points using the same set of genes. It was also found that this abnormally sulfated keratan sulfate is not associated with the collagen fibrils which eventually results in the loss of corneal transparency. ${ }^{24}$ These studies explain the pathological symptoms of MCD-affected cornea, but the mechanism through which these alterations happen from the normal healthy physiology are not well understood. The present study is focused on observing the mechanisms through which the transparency and the allied anatomical defects occur in the MCD-affected corneas.

PAPS is the universal sulfonate donor for all the membrane associated and cytosolic sulfotransferase reactions. ${ }^{25}$ 3'-phosphoadenosine 5'-phosphosulfate synthase forms PAPS in a two-step process. In the first step adenosine 5 '-phosphosulfate (APS) and pyrophosphate are formed by combining ATP with inorganic phosphate in a process catalyzed by ATP sulfurylase domain of the enzyme. In the 
a

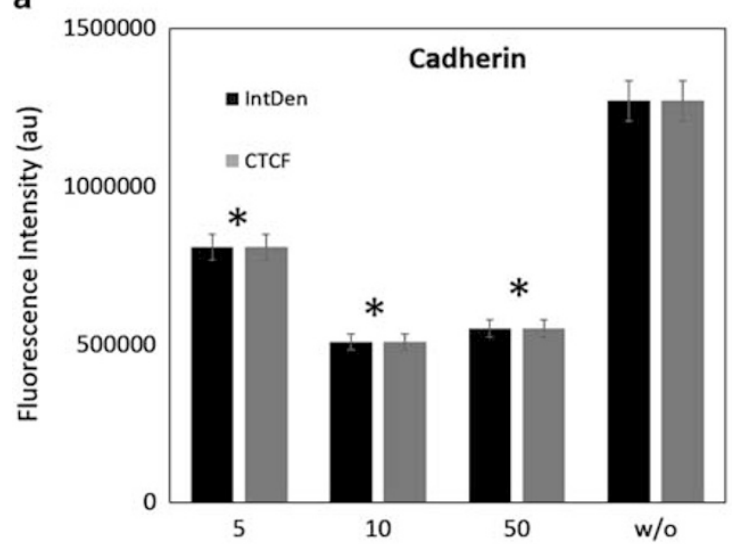

c

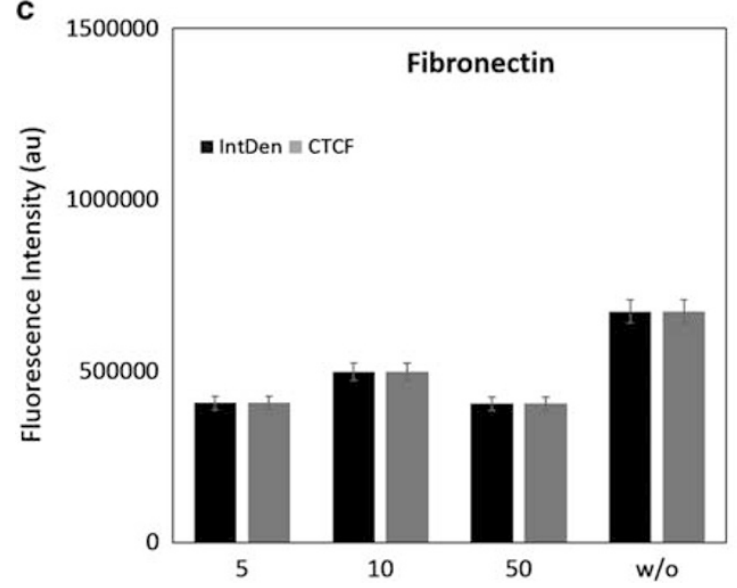

b

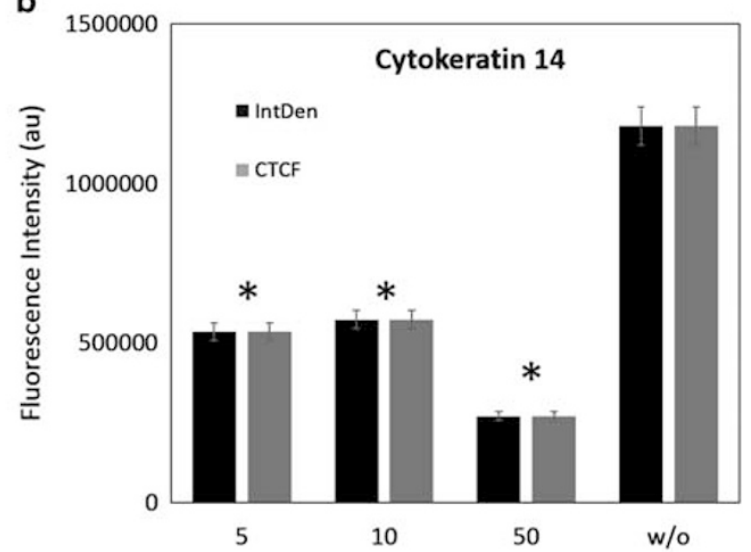

d

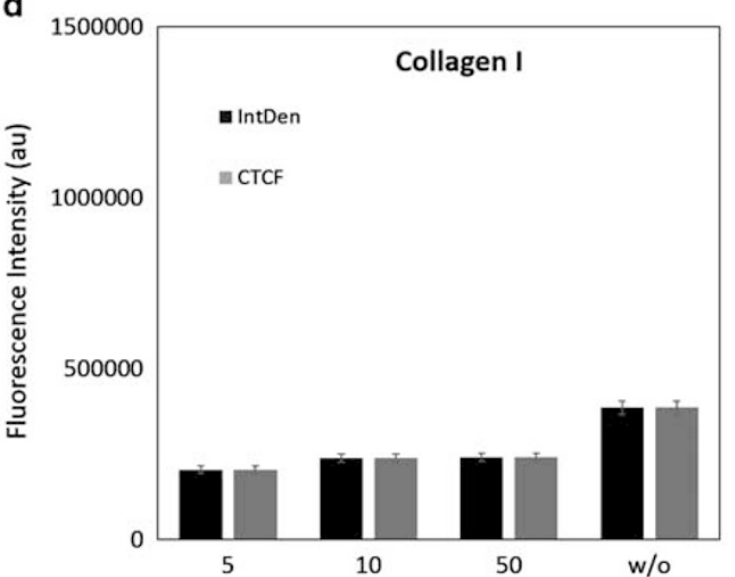

Figure 8 Quantification of cell fluorescence using ImageJ, where IntDen and CTCF represent integrated density and corrected total cell fluorescence, respectively for (a) Cadherin, (b) Cytokeratin 14, (c) Fibronectin, and (d) Collagen type I (statistical significance between tests and control: ${ }^{*} P<0.001$, $n=3)$.

second step, PAPS is formed by combining an ATP molecule with APS, during which ADP is generated as a byproduct by the APS kinase domain. ${ }^{25}$ Sodium chlorate is a competitive inhibitor of the ATP sulfurylase activity of PAPSS enzyme; thus it inhibits the formation of PAPS which is the sole donor of sulfonate and thus ceases the process of sulfation in the cells. This includes both the tyrosine sulfation of proteins and carbohydrate residues that form proteoglycans. ${ }^{26}$ Sulfation is a low capacity and high affinity enzymatic process, which involves high consumption of PAPS thus making the PAPS synthesis process a rate limiting step in the cascade reaction. Thus in order to inhibit the sulfation of keratan sulfate molecules to mimic the diseased condition of MCD, sodium chlorate was chosen as it is an efficient inhibitor of PAPS synthetase and in the absence of a sulfonate donor, the sulfation process of keratan sulfate is automatically hindered. The concentrations of sodium chlorate chosen for inhibiting sulfation of keratan sulfate were based on earlier studies demonstrating the effect of this molecule on the inhibition of sulfation. ${ }^{27}$

Thus as a first step to develop an in vitro model of MCD we envisaged of culturing corneal stromal cells in monolayer, which are indulged in production of abnormally sulfated keratan sulfate molecules. A range of sodium chlorate concentration was chosen to identify the quantitative effect of sodium chlorate on the inhibition of sulfation mechanism. Endothelial cells, which are also known to produce abnormally sulfated keratan sulfate, were not included in this model to keep it simple, as a first step toward development of complete model of the disease in vitro. The decreased metabolic activity of the cells as a function of increasing concentration of sodium chlorate indicated that the addition of sodium chlorate inhibits the sulfation processes in the cytosol. But in the second week the metabolic activity of the cells recovered up to the baseline; without any evident effect of sodium chlorate. This elucidates that the cellular metabolism acclimatize with time to the presence sodium chlorate, may be by modifying the kinetics of the ATP sulfurylase activity of PAPS synthetase enzyme so that the basic metabolic functions of the cell are not affected. DNA quantification at day 14, indicated no effect of sodium chlorate on cellular proliferation of corneal stromal cells. Earlier reports showed that long term exposure to sodium chlorate does not have significant effect on cellular proliferation in case of MG-63 cells (osteosarcoma cell line). ${ }^{10}$ 

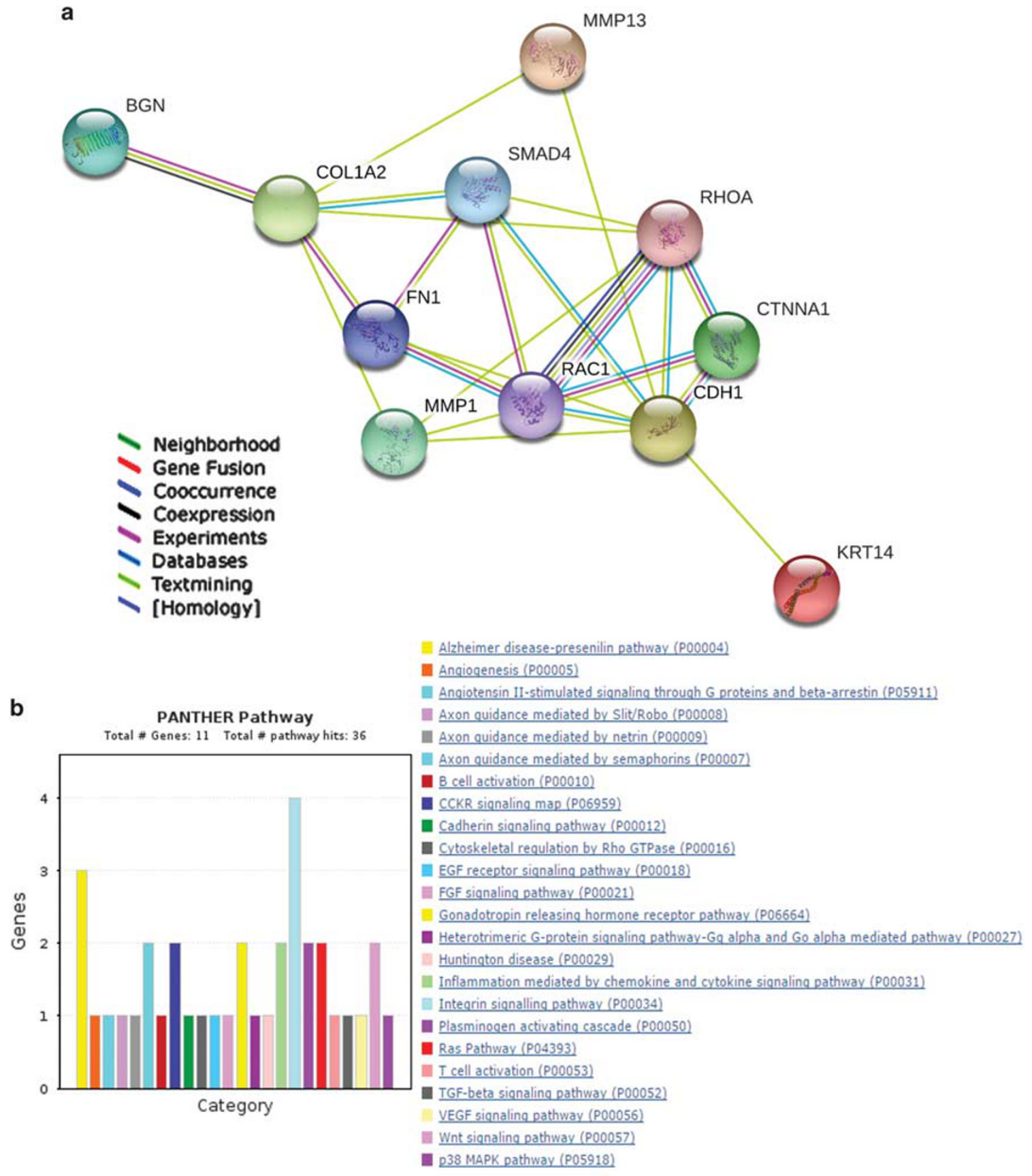

Figure 9 Protein-protein interaction studies carried out with the genes studied and found affected by the administration of sodium chlorate to corneal stromal cells. It was observed that the $\beta$-CTNN gene formed the biggest subnetwork with highest protein interactions taking place from this hub. Further, the gene ontological studies demonstrated the role of WNT, integrin, cadherin, and cytoskeletal signaling pathways in the disease progression of MCD.

However, the morphological studies showed prominent effect of sodium chlorate on corneal stromal cells. Stressed cellular morphology may be due to the effect of this inhibitor on cellular cytoskeleton and focal adhesion point formation. This observation is in direct contrast to the earlier report on MG-63 cells, which were shown to have no effect of sodium chlorate concentration on cellular morphology even on longterm exposure. ${ }^{10}$ It could be due to the difference in response of different cell types to inhibition of sulfation.
Sulfated GAGs have a crucial role in guiding cellular morphology. GAGs are reported to modulate the biochemical properties of cell adhesion molecules like fibronectin. ${ }^{28}$ It was further elucidated that in presence of increased amount of sulfated GAGs; HeLa and HT-1080 cells doubled their surface areas. Sodium chlorate also affects the sulfation of GAGs so the altered morphology of the cells could be a result of this alteration. ${ }^{28}$ As the sulfation is hindered by sodium chlorate, the surface area of corneal stromal cells reduced as a function 


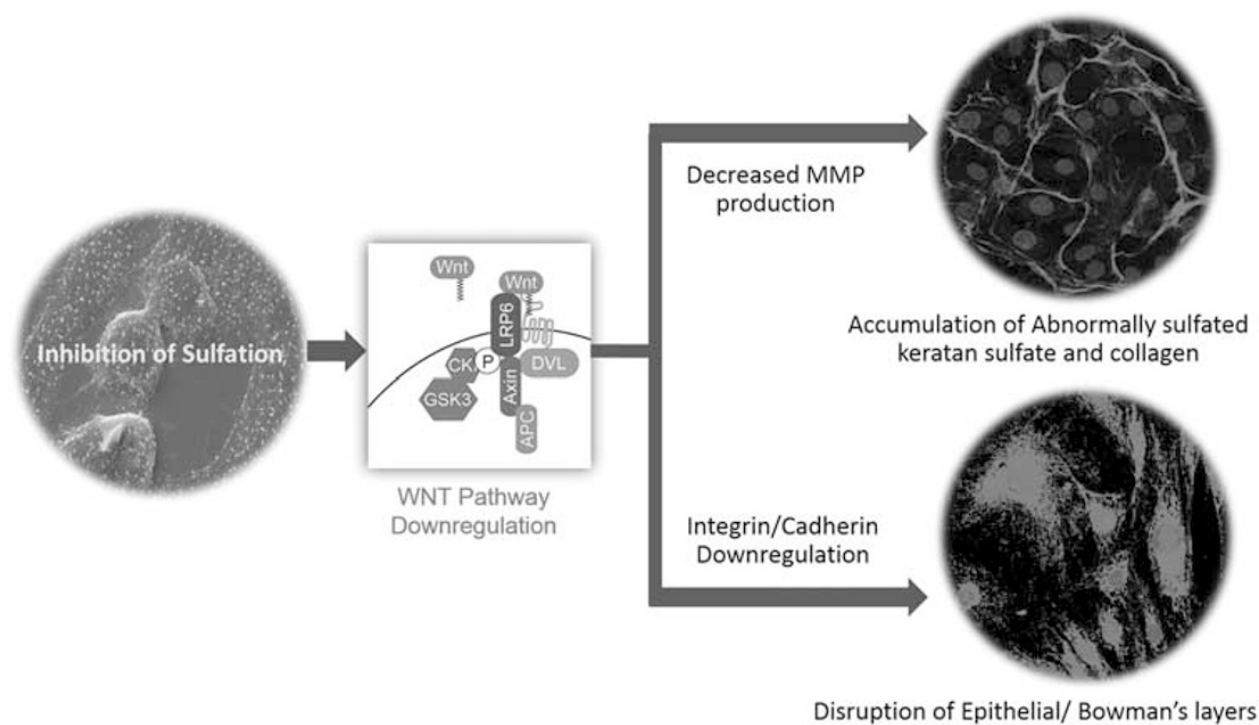

Figure 10 Schematic of the MCD disease progression pathway as elucidated by the current study. The inhibition of sulfation of keratan sulfate results into the downregulation of WNT pathway that leads to downregulation of two protein groups. The first one includes cadherins and integrins that help in cell-cell and cell-matrix interactions. In the absence of these proteins, the epithelium membrane and thus the Descemet's membrane gets disrupted. But the second group involves MMPs. In the absence of MMPs, the abnormally sulfated keratan sulfate and thickened collagen bundles could not be degraded and thus accumulate in the stroma resulting in the loss of transparency of the tissue over time.

of its increasing concentration. Syndecans, a family of transmembrane heparan sulfate proteoglycans, regulate cellular morphology in mammary epithelial tumor cells. ${ }^{29}$ Introduction of a normal copy of syndecan gene in the tumor cells could recover their normal epithelial cell morphology. ${ }^{29}$ This indicates that any alteration in the sulfated GAGs may cause a change in cellular morphology directly. Though in the present study we investigated the effect of abnormal sulfation of keratan sulfate; but the effect of abnormal sulfation of other proteoglycans caused by the sulfation inhibitor cannot be avoided. The total sulfated GAG content as observed in the biochemical estimation; reduced as a function of increasing concentration of sodium chlorate in the test groups, validating the use of this PAPS synthetase inhibitor in creating MCD disease model. Raman microspectroscopy was to confirm the biochemical studies and the keratan sulfate deposits seen in the SEM images, which confirmed the presence of keratan sulfate in all the samples and the decreasing amount of sulfate with the increasing concentration of sodium chlorate. Interestingly, the total collagen content also reduced with the increasing concentration of sodium chlorate in the corneal stromal cells. Corneal stroma mainly contains collagen type I, thus indicating that sodium chlorate has a negative effect of expression of collagen type I. Earlier it was shown that sodium chlorate enhances expression of collagen type II in chondrocytes. ${ }^{9}$

For gene expression studies, three sets of genes were chosen; related to cellular adhesion, extracellular matrix modelling and signaling pathway for ECM production. These three set of genes were chosen to assess the effect of inhibition of keratan sulfate sulfation in generating the hallmark pathological symptoms of MCD. Gene expression studies demonstrated a direct negative effect of sodium chlorate on the expression of: extracellular matrix proteins (biglycan and collagen type I), matrix remodeling enzymes (MMP I and MMP 13), focal adhesion proteins (RAC 1 and RHO A), cellular adhesion proteins (fibronectin and E-cadherin), and corneal stromal cell marker (Cytokeratin 14). The expression of Cytokeratin 14 reduced with the increasing concentration of sodium chlorate, indicating a dedifferentiation or loss of corneal stromal phenotype in the cells. ${ }^{30}$ Although the control group of cells expressed high levels of expression for the marker indicating their normal phenotype. Expression of extracellular matrix proteins was also negatively affected by the increasing concentration of sodium chlorate indicating the effect of poor sulfation on the expression of these genes including biglycan and collagen type I. Biglycan is a small leucine rich proteoglycan (SLRP) whose expression is generally increased in diseased conditions of cornea such as bullous keratopathy, chronic edema and keratoconus. ${ }^{31}$ But the sulfation inhibition in the present model of MCD caused a decrease in its expression with the increasing concentration of the inhibitor. This indicates that production of all sulfated GAGs including SLRPs is negatively affected by inhibition of sulfation. This also validated the results from biochemical estimations that indicated a decrease in total sulfated GAG content, with the increasing inhibitor concentration. Collagen type I gene expression further validated the biochemical estimation that inhibition of sulfation causes a detrimental effect on its expression. Catabolic activities are also equally important for the dynamic remodeling of the corneal tissue, thus the gene expression of MMP 1 and MMP 13 which are 
matrix metalloproteinases involved in collagen type I degradation was studied. Both the genes are downregulated as compared with the control group expressions showing negative effect of the inhibitor on their expressions too. This is a very important finding regarding the progression of $\mathrm{MCD}$ as both the abnormally thick collagen bundles and abnormally sulfated proteoglycans; mainly keratan sulfate gets accumulated in the diseased tissue. This can be a direct implication of the downregulation of these matrix remodeling enzymes which helps in degradation of both collagen and proteoglycans. MMP 1 (interstitial collagenase) is involved in the degradation of all extracellular matrix proteins including collagen type I. It has been reported that MMP 1 activity is essential for the remodeling and migration of corneal stromal cells in the tissue. ${ }^{32}$ But MMP 13 (collagenase III) is involved in the degradation of collagen type I. MMP 13 has been reported to be involved in corneal remodeling after injury, indicating its requirement for the remodeling process. ${ }^{33}$ Thus, downregulation of MMP expression caused by the inhibition of sulfation leads to accumulation of both abnormally sulfated keratan sulfate and abnormally thick collagen bundles in the corneal stroma. These abnormal accumulations in the stroma finally lead to loss of corneal transparency.

Expression of RHO A and RAC 1 which are small $G$ protein signaling molecules involved in integrin-mediated cellsubstratum attachment ${ }^{34}$ was also downregulated by inhibition of sulfation in the corneal stromal cells. RHO A showed concentration-dependent downregulation but RAC 1 showed a concentration-independent downregulation. RHO A and RAC 1 are both involved in the formation of integrin complexes that form the focal adhesion points where the cells bind to fibronectin like ligands of the extracellular matrix and induce cytoskeletal changes upstream that guide the cellular morphology after attachment to the substratum. The downregulation of these genes would directly result in the nonformation of focal complexes and thus poor attachment of the cells, which can explain the initial rounded morphology of the corneal stromal cells in first week and later the stressed morphologies in second week. Fibronectin helps in cellular attachment through integrin-mediated adhesion mechanism. ${ }^{35}$ It was also downregulated as an effect of decreased sulfation in the cells, while cadherin which is responsible for the formation of cell to cell junctions ${ }^{36}$ was also downregulated due to the inhibition of sulfation in the MCD model tests. It has earlier been reported that inhibition of sulfation causes downregulation of $\mathrm{N}$-cadherin and fibronectin during chondrogenesis. ${ }^{9}$ All these results can explain the pathological symptom of disrupted epithelial and bowman's layer in the MCD-affected corneas. As all the cellcell and cell-matrix adhesion molecules are downregulated resulting in tissue disruption; as a result of the abnormal sulfation of keratan sulfate.

Finally, to assess through which signaling pathways these gene expressions are being modulated we checked for the expression of SMAD 4, which is involved in TGF $\beta$-SMAD pathway and $\beta$-catenin, which is involved in WNT pathway. SMAD 4 showed a concentration-independent inhibition due to sodium chlorate while $\beta$-catenin showed a concentrationdependent inhibition. This indicated that both the pathways are negatively affected because of inhibition of sulfation. GAG sulfation has a crucial role in WNT signaling, an alteration in sulfation of GAGs causes diminished response to WNT signaling in articular chondrocytes. ${ }^{37}$ WNT pathway is known to have a crucial role in cellular adhesion through cadherins and intergrins as also found in the protein-protein interaction studies. ${ }^{38,39}$ Further, WNT pathway is known to involve in the induction of MMP expression. ${ }^{40,41}$ Thus, the downregulation of WNT pathway by decreased sulfation of GAGs, directly results in decreased expression of MMPs, which was also validated by the gene interaction studies that showed close interaction between these genes. The gene ontological analysis further indicated the major role of WNT pathway in the disease progression of MCD (Figure 9). Subsequently, the reduced expression of MMP enzymes results into the accumulation of abnormally sulfated GAGs and thick collagen bundles in the corneal stroma as they cannot be degraded. This ultimately results into progressive loss of corneal transparency because of these accumulations. Thus therapies helping in enhancing MMP production in MCD affected corneas or direct delivery of these enzymes into the MCDaffected corneas in the patients can be tested a potential therapy for the treatment of MCD.

Thus, the present study for the first time elucidated the mechanism of deterioration of epithelial and Bowman's layer of cornea, which is caused due to inhibition of integrin- and cadherin-mediated cell-cell and cell-matrix interactions through deregulation of WNT pathway. The second and main pathological sign of the disease; that is, accumulation of abnormally sulfated keratan sulfate and thick collagen fibers is caused due to the reduced production of MMP enzymes again due to suppression of WNT pathway. Both these conditions are a direct result of inhibition of sulfation of keratan sulfate in the corneal stromal cells. Thus, this in vitro model of MCD could elucidate onset of the two most important pathological features of MCD. The current model has its own limitations, such as monolayer culture system, non-specific inhibition of GAG sulfation. In addition, we generated insights about expression of specific genes only; while other genes might also have important roles in the pathophysiology of the disease.

This study is a first step toward establishment of a biomimetic in vitro disease model of MCD. Further studies are needed to acquire knowledge of other genes that can play a part in the onset and progression of the disease. As next step, establishment of a 3D in vitro tissue engineered model using 3D bioprinting technique ${ }^{42,43}$ would help to simulate the layered corneal structure with different cell types simulating endothelial, stromal, and epithelial cell layers of MCD-affected cornea. The study also highlights that two important family of proteins MMPs and integrins/cadherins need to be targeted for development of therapies for treating 
MCD. One strategy can be the direct delivery/administration of MMPs into the MCD-affected corneas to dissolve the opacities as they occur during the progression of the disease, thus paving a way to cure MCD caused corneal blindness.

\section{ACKNOWLEDGMENTS}

This study was supported by intramural funding from IIT Delhi (High Impact project) and funding from Department of Biotechnology, Government of India (BT/PR5717/MED/32/244/2012 and BT/MB/Indo-US/VR/09/2013).

\section{DISCLOSURE/CONFLICT OF INTEREST}

The authors declare no conflict of interest.

1. Musch DC, Niziol LM, Stein JD, et al. Prevalence of corneal dystrophies in the United States: Estimates from claims data. Investig Ophthalmol Vis Sci 2011;52:6959-6963.

2. Snip RC, Kenyon KR, Green WR. Macular corneal dystrophy: ultrastructural pathology of corneal endothelium and Descemet's membrane. Invest Ophthalmol 1973;12:88-97.

3. Cheng J, Qi X, Zhao J, et al. Comparison of penetrating keratoplasty and deep lamellar keratoplasty for macular corneal dystrophy and risk factors of recurrence. Ophthalmology 2013;120:34-39.

4. Akama TO, Nishida K, Nakayama J, et al. Macular corneal dystrophy type I and type II are caused by distinct mutations in a new sulphotransferase gene. Nat Genet 2000;26:237-241.

5. Cheng $X$, Pinsky PM. Mechanisms of self-organization for the collagen fibril lattice in the human cornea. J R Soc Interface 2013;10:20130512.

6. Knupp C, Pinali C, Lewis PN, et al. The architecture of the cornea and structural basis of its transparency. Adv Protein Chem Struct Biol 2009;78: 25-49.

7. Farrell RA, Hart RW. On the theory of the spatial organization of macromolecules in connective tissue. Bull Math Biophys 1969;31:727-760.

8. Müller LJ, Pels E, Schurmans LRHM, et al. A new three-dimensional model of the organization of proteoglycans and collagen fibrils in the human corneal stroma. Exp Eye Res 2004;78:493-501.

9. Cho YR, Lee SJ, Jeon HB, et al. Under-sulfation by PAPS synthetase inhibition modulates the expression of ECM molecules during chondrogenesis. Biochem Biophys Res Commun 2004;323:769-775.

10. Kumarasuriyar A, Lee I, Nurcombe V, et al. De-sulfation of MG-63 cell glycosaminoglycans delays in vitro osteogenesis, up-regulates cholesterol synthesis and disrupts cell cycle and the actin cytoskeleton. J Cell Physiol 2009;219:572-583.

11. Greve H, Cully Z, Blumberg P, et al. Influence of chlorate on proteoglycan biosynthesis by cultured human fibroblasts. J Biol Chem 1988;263:12886-12892.

12. Safaiyan F, Kolset SO, Prydz K, et al. Selective effects of sodium chlorate treatment on the sulfation of heparan sulfate. J Biol Chem 1999;274: 36267-36273.

13. Chameettachal $\mathrm{S}$, Murab $\mathrm{S}$, Vaid $\mathrm{R}$, et al. Effect of visco-elastic silk-chitosan microcomposite scaffolds on matrix deposition and biomechanical functionality for cartilage tissue engineering. J Tissue Eng Regen Med 2015.

14. Nara S, Chameettachal S, Midha S, et al. Preservation of biomacromolecular composition and ultrastructure of a decellularized cornea using a perfusion bioreactor. RSC Adv 2016;6:2225-2240.

15. Murab S, Samal J, Shrivastava A, et al. Glucosamine loaded injectable silk-in-silk integrated system modulate mechanical properties in bovine ex-vivo degenerated intervertebral disc model. Biomaterials 2015;55:64-83.

16. Murab S, Chameettachal S, Bhattacharjee $M$, et al. Matrix-embedded cytokines to simulate osteoarthritis-like cartilage microenvironments. Tissue Eng Part A 2013;19:1733-1753.

17. Bhattacharjee $M$, Chawla S, Chameettachal S, et al. Role of chondroitin sulphate tethered silk scaffold in cartilaginous disc tissue regeneration. Biomed Mater 2016;11:025014.

18. Abedin MN, Bradley AT, Sharma SK, et al. Mineralogy and astrobiology detection using laser remote sensing instrument. Appl Opt 2015;54: 7598-7611.
19. Tremblay M, Dubé I. Macular dystrophy of the cornea. Ultrastructure of two cases. Can J Ophthalmol 1973;8:47-53.

20. Klintworth GK. Current concepts on the ultrastructural pathogenesis of macular and lattice corneal dystrophies. Birth Defects Orig Artic Ser 1971;7:27-31.

21. Meek KM, Quantock AJ, Elliott GF, et al. Macular corneal dystrophy: the macromolecular structure of the stroma observed using electron microscopy and synchrotron X-ray diffraction. Exp Eye Res 1989;49: 941-958.

22. Quantock AJ, Meek KM, Ridgway AE, et al. Macular corneal dystrophy: reduction in both corneal thickness and collagen interfibrillar spacing. Curr Eye Res 1990;9:393-398.

23. Palka BP, Sotozono $\mathrm{C}$, Tanioka $\mathrm{H}$, et al. Structural collagen alterations in macular corneal dystrophy occur mainly in the posterior stroma. Curr Eye Res 2010;35:580-586.

24. Lewis D, Davies Y, Nieduszynski IA, et al. Ultrastructural localization of sulfated and unsulfated keratan sulfate in normal and macular corneal dystrophy type I. Glycobiology 2000;10:305-312.

25. Venkatachalam KV. Human 3'-phosphoadenosine 5'-phosphosulfate (PAPS) synthase: biochemistry, molecular biology and genetic deficiency. IUBMB Life 2003;55:1-11.

26. Baeuerle PA, Huttner WB. Chlorate-a potent inhibitor of protein sulfation in intact cells. Biochem Biophys Res Commun 1986;141:870-877.

27. Keller KE, Bradley JM, Kelley MJ, et al. Effects of modifiers of glycosaminoglycan biosynthesis on outflow facility in perfusion culture. Invest Ophthalmol Vis Sci 2008:49:2495-2505.

28. Klebe RJ, Escobedo L V, Bentley KL, et al. Regulation of cell motility, morphology, and growth by sulfated glycosaminoglycans. Cell Motil Cytoskeleton 1986;6:273-281.

29. Leppä S, Mali M, Miettinen HM, et al. Syndecan expression regulates cell morphology and growth of mouse mammary epithelial tumor cells. Proc Natl Acad Sci USA 1992;89:932-936.

30. Nieto-Miguel T, Calonge M, de la Mata A, et al. A comparison of stem cell-related gene expression in the progenitor-rich limbal epithelium and the differentiating central corneal epithelium. Mol Vis 2011;17: 2102-2117.

31. Funderburgh JL, Hevelone ND, Roth MR, et al. Decorin and biglycan of normal and pathologic human corneas. Invest Ophthalmol Vis Sci 1998:39:1957-1964.

32. Daniels JT, Limb GA, Saarialho-Kere U, et al. Human corneal epithelial cells require MMP-1 for HGF-mediated migration on collagen I. Invest Ophthalmol Vis Sci 2003;44:1048-1055.

33. Ye $H Q$, Maeda $M, Y u ~ F S$, et al. Differential expression of MT1-MMP (MMP-14) and collagenase III (MMP-13) genes in normal and wounded rat corneas. Invest Ophthalmol Vis Sci 2000;41: 2894-2899.

34. Fukata $\mathrm{M}$, Nakagawa $\mathrm{M}$, Kuroda $\mathrm{S}$, et al. Cell adhesion and Rho small GTPases. J Cell Sci 1999;112(Pt 2):4491-4500.

35. Pankov R. Fibronectin at a glance. J Cell Sci 2002;115:3861-3863.

36. Van Roy F, Berx G. The cell-cell adhesion molecule E-cadherin. Cell Mol Life Sci 2008;65:3756-3788.

37. Shortkroff S, Yates KE. Alteration of matrix glycosaminoglycans diminishes articular chondrocytes' response to a canonical Wnt signal. Osteoarthritis Cartilage 2007;15:147-154.

38. Wu B, Crampton SP, Hughes CCW. Wht signaling induces matrix metalloproteinase expression and regulates $\mathrm{T}$ cell transmigration. Immunity 2007;26:227-239.

39. Lowy AM, Clements WM, Bishop J, et al. beta-Catenin/Wnt signaling regulates expression of the membrane type 3 matrix metalloproteinase in gastric cancer. Cancer Res 2006;66:4734-4741.

40. Amin N, Vincan E. The Wnt signaling pathways and cell adhesion. Front Biosci (Landmark Ed 2012;17:784-804.

41. Heuberger J, Birchmeier W. Interplay of cadherin-mediated cell adhesion and canonical Wnt signaling. Cold Spring Harb Perspect Biol 2010;2:a002915.

42. Chameettachal S, Midha S, Ghosh S. Regulation of chondrogenesis and hypertrophy in silk fibroin-gelatin based 3D bioprinted constructs. ACS Biomater Sci Eng 2016;2:1450-1463.

43. Nara S, Chameettachal S, Ghosh S. Precise patterning of biopolymers and cells by direct write technique. Mater Technol 2014;29: B10-B14. 